\title{
Reproductive endocrine effects of intranasal administration of norethisterone to adult female rhesus monkeys (Macaca mulatta)*
}

\author{
V. Puri, G. F. X. David, A. K. Dubey, C. P. Puri† and T. C. Anand Kumar $†$ \\ Experimental Biology Unit, Department of Anatomy, WHO-CCR, All India Institute of Medical \\ Sciences, New Delhi 110 029, India
}

\begin{abstract}
Summary. Intranasal administration of norethisterone at a daily dose of $9 \mu \mathrm{g}$ between Days 5 and 14 of the menstrual cycles blocked ovulation in 10 out of 17 adult female monkeys. Serum concentrations of hormones indicated that ovulation was blocked due to a suppression of the mid-cycle, oestradiol-induced LH surge. Ovarian follicular activity in the treated menstrual cycles was not affected by norethisterone but there was a marked delay in the onset of the mid-cycle oestradiol surge in most of the treated animals. The duration of the menstrual cycle length after the oestradiol peak was significantly reduced in all the treated monkeys, indicative of a shortened luteal phase.
\end{abstract}

\section{Introduction}

The bioavailability of steroids is significantly enhanced when administered intranasally as compared with their systemic administration (Anand Kumar, David, Umberkoman \& Saini, 1974; Anand Kumar, David, Sankaranarayanan, Puri \& Sundaram, 1982). The difference in the pharmacokinetic patterns between the two routes of administration has been related to the different pathways and the rates at which the steroid enters the general circulation from the site of administration (Anand Kumar et al., 1982). As a consequence of the increased bioavailability, the intranasal route offers a potential advantage over the conventionally used oral or systemic routes in terms of lowering the dose of the administered steroids without concomitant reduction in their pharmacological efficacy (Anand Kumar, David, Kumar, Umberkoman \& Krishnamoorthy, 1976). Such an advantage could be exploited in the administration of contraceptive steroids which are currently being used by a large number of individuals quite extensively. Studies on the efficacy of steroids administered intranasally have been undertaken with female (Anand Kumar, David \& Puri, 1977) and male (Anand Kumar, Sehgal, David, Bajaj \& Prasad, 1980a) rhesus monkeys. The ability of norethisterone to inhibit ovulation was the only criterion used to evaluate the efficacy of the intranasal route and these studies showed that ovulation was not uniformly inhibited (Anand Kumar et al., 1977). A more comprehensive study carried out recently on women treated with a minipill of $300 \mu \mathrm{g}$ norethisterone by Landgren \& Diczfalusy (1980) showed that inhibition of ovulation is not the only way in which the steroid acts; there was inhibition of follicular and luteal activities, occurrence of normal follicular activity but absence of luteal function, poor luteal function and some women did not show any differences in their ovarian activities.

In the present investigations with rhesus monkeys, the effects of norethisterone were studied with particular reference to circulating concentrations of oestradiol and progesterone in blood samples taken throughout the treatment menstrual cycle and concentrations of bioactive luteinizing hormone $(\mathrm{LH})$ on the days around the mid-cycle oestradiol peak.

* Reprint requests to Dr T. C. Anand Kumar.

$\dagger$ Present address: WHO-CCR, Institute for Research in Reproduction, Parel, Bombay 400 012, India. 


\section{Materials and Methods}

Animals. Healthy parous, female rhesus monkeys ( $\mathrm{N}=27$ ), each weighing $5-6 \mathrm{~kg}$, were taken from the Primate Research Facility of the Institute. The studies were carried out between September and April when the incidence of ovulatory menstrual cycles is maximal in this colony (Anand Kumar et al., 1980b). Only animals exhibiting at least two consecutive ovulatory menstrual cycles of normal duration (23-31 days), as determined by laparoscopy and examination of daily vaginal smears, were taken for the studies.

Reagents. All the reagents used were of analytical grade. Norethisterone (Steraloids, Inc., Wilton, NH, U.S.A.), oestradiol and progesterone (Sigma Chemicals, St Louis, MO, U.S.A.) were used without further purification. The trititum-labelled steroids were obtained from the Radiochemical Centre, Amersham, U.K., and were checked for their radiochemical purity by thin-layer chromatography before use.

The antisera for oestradiol, progesterone and testosterone were supplied by the World Health Organization under their quality control programme for radioimmunoassay methods (Hall, 1978). Rhesus monkey pituitary extract (LER-1909-2; National Institutes of Health, U.S.A.) was used as the reference standard for estimating $\mathrm{LH}$.

Treatment. Norethisterone was dissolved in a solution of propylene glycol:ethanol:water (3:3:4 by vol.) and was sprayed intranasally using a precalibrated glass atomizer as described previously (Gopinath, Gopinath \& Anand Kumar, 1978; Anand Kumar et al., 1979). The animals were treated between 10:00 and 11:00 h with nasal sprays on Days 5-14 (Day 1 is the first day of menstruation) of the menstrual cycle. The control monkeys $(\mathrm{N}=10)$ were sprayed with the solvent only whereas the experimental animals $(N=17)$ were sprayed with a daily dose of $9 \mu \mathrm{g}$ norethisterone.

Monitoring of menstrual cycles. The length of the menstrual cycle and duration of menses were monitored by daily microscopic examination of vaginal smears.

Laparoscopy. The animals were sedated with ketamine hydrochloride $(10 \mathrm{mg} / \mathrm{kg}$ body weight, i.m.) and laparoscopy was performed on Day 20 of the menstrual cycle during the pretreatment and treatment cycles to determine whether ovulation had occurred, as indicated by the presence of a newly formed corpus luteum.

Collection of blood samples. Blood samples $(5 \mathrm{ml}$ each) were taken by syringe from the saphenous vein daily throughout the menstrual cycles, between 10:00 and 11:00 h before treatment. Serum was separated and stored at $-20^{\circ} \mathrm{C}$ until assayed for various hormones.

Assay of hormones. Concentrations of oestradiol and progesterone in the serum samples were measured by specific radioimmunoassay (RIA) methods (Anand Kumar et al., 1980c). The sensitivities of the assays were $10 \mathrm{pmol} / 1$ for oestradiol and $15 \mathrm{pmol} / 1$ for progesterone. The coefficients of variation within and between assays in the present study were $3.2 \%$ and $7.09 \%$ for oestradiol $(\mathrm{N}=25)$ and $3.8 \%$ and $6.3 \%$ for progesterone $(\mathrm{N}=30)$, respectively. Concentrations of LH were estimated by an in-vitro bioassay method using Leydig cells from mouse testes (Puri, Puri, David \& Anand Kumar, 1980). LH was measured in every sample taken from the solvent-treated controls, but only in those taken 2 days before, on the day of and 2 days after the mid-cycle oestradiol peak in the norethisterone-treated females. The sensitivity of the LH assay was $2 \mathrm{ng}$ LER-1909-2 equiv./tube and the coefficients of variation within and between assays were $9 \cdot 3 \%$ and $11 \cdot 3 \%$ $(\mathrm{N}=19)$ respectively.

The RIA data were analysed by logit-log transformation to obtain a linear dose-response curve. followed by unweighted or weighted least square regression analysis using a Hewlett-Packard calculator (Model 9831). 
Evaluation of endocrine effects. The most consistent endocrine feature of all the menstrual cycles studied was the occurrence of the 'mid-cycle' preovulatory oestradiol peak; this was taken as the cardinal point to divide the menstrual cycle into two phases. Phase I included Day 1 of menstruation upto and inclusive of the day of the 'mid-cycle' oestradiol peak. Phase II included the day after the oestradiol peak to the penultimate day of the ensuing menstruation. These two phases are not synonymous with the follicular and luteal phases of the cycle because ovulation is known to occur in monkeys $36-40 \mathrm{~h}$ (Weick et al., 1973) after the oestradiol-induced LH surge and therefore Phase II of the menstrual cycle would also include a small part of the follicular phase.

Statistical test. The mean oestradiol peak values at mid-cycle and the mean progesterone concentrations during Phase II of the menstrual cycles of controls and norethisterone-treated monkeys were compared by Student's paired $t$ test. The concentration of LH on the 2 days before, on the day of and on 2 days after the 'mid-cycle' oestradiol peak were also similarly compared by Student's paired $t$ test. The day of the mid-cycle oestradiol peak was compared between treated and control females to determine whether there was any temporal shift in the day of the mid-cycle oestradiol peak. The duration of Phase II of the menstrual cycle between the treated and controls was also studied and compared by Student's paired $t$ tests.

\section{Results}

Menstrual cycles

All 10 solvent-treated controls exhibited ovulatory menstrual cycles and the mean cycle length was about 26 days during the pretreatment, treatment and post-treatment periods (Table 1). Menstruation in the controls was of normal duration (3-5 days).

Of the 17 norethisterone-treated animals, 7 showed ovulatory menstrual cycles which were of normal duration and the remaining 10 showed anovulatory cycles. The cycle length in 4 of these 10 anovulatory monkeys was significantly $(P<0.01)$ reduced to about 17 days while in the other 6 anovulatory animals the cycle length was not different as compared with the controls. For convenience of description the 4 anovulatory animals showing shortened cycles were considered as Group I and the 6 anovulatory animals showing cycles of normal duration are included in Group II. The post-treatment cycle length in some of the anovulatory animals was prolonged (Table 1).

Menstruation in all the norethisterone-treated animals was of normal duration during the treatment and the post-treatment cycles.

Table 1. Menstrual cycle characteristics in female rhesus monkeys treated with norethisterone nasal sprays

\begin{tabular}{|c|c|c|c|c|c|c|}
\hline Treatment & $\begin{array}{l}\text { No. of } \\
\text { females }\end{array}$ & $\begin{array}{l}\text { Pretreatment } \\
\text { cycle length } \\
\text { (days) }\end{array}$ & $\begin{array}{l}\text { Treatment } \\
\text { cycle length } \\
\text { (days) }\end{array}$ & $\begin{array}{c}\text { Day of } \\
\text { oestradiol peak } \\
\text { in treatment } \\
\text { cycle }\end{array}$ & $\begin{array}{l}\text { Length of } \\
\text { Phase II of } \\
\text { treatment cycle } \\
\text { (days) }\end{array}$ & $\begin{array}{l}\text { Post-treatment } \\
\text { cycle length } \\
\text { (days) }\end{array}$ \\
\hline $\begin{array}{l}\text { None (controls) } \\
\text { Norethisterone }\end{array}$ & 10 & $25 \cdot 9 \pm 1 \cdot 6$ & $26 \cdot 2 \pm 2 \cdot 3$ & $9 \cdot 0 \pm 1 \cdot 2$ & $17 \cdot 4 \pm 1 \cdot 4$ & $26 \cdot 6 \pm 2 \cdot 8$ \\
\hline $\begin{array}{l}\text { Norethisterone } \\
\text { Anovulatory cycles }\end{array}$ & & & & & & \\
\hline Group I & 4 & $\begin{array}{c}25 \cdot 2 \pm 3 \cdot 6 \\
(20-28)\end{array}$ & $\begin{array}{l}17 \cdot 2 \pm 2 \cdot 4^{* * *} \\
(14-19)\end{array}$ & $9 \cdot 2 \pm 0 \cdot 9$ & $8 \cdot 0 \pm 1 \cdot 4 * * *$ & $\begin{array}{l}52 \cdot 0 \pm 25 \cdot 1 \\
(24-77)\end{array}$ \\
\hline Group II & 6 & $\begin{array}{c}25 \cdot 7 \pm 1 \cdot 0 \\
(24-27)\end{array}$ & $\begin{array}{c}27 \cdot 2 \pm 1 \cdot 6 \\
(24-28)\end{array}$ & $12 \cdot 0 \pm 1 \cdot 8^{* * * *}$ & $15 \cdot 2 \pm 1 \cdot 7^{*}$ & $\begin{array}{c}32 \cdot 2 \pm 9 \cdot 7 \\
(25-46)\end{array}$ \\
\hline Ovulatory cycles & 7 & $\begin{array}{c}26 \cdot 3 \pm 4 \cdot 3 \\
(21-33)\end{array}$ & $\begin{array}{c}26 \cdot 3 \pm 4 \cdot 3 \\
(21-33)\end{array}$ & $11 \cdot 5 \pm 2 \cdot 8^{* *}$ & $14 \cdot 7 \pm 1 \cdot 8^{* * *}$ & $\begin{array}{c}28 \cdot 0 \pm 4 \cdot 1 \\
(25-37)\end{array}$ \\
\hline
\end{tabular}

Values are mean \pm s.d.; values in parentheses are ranges.

${ }^{*} P<0.01,{ }^{* *} P<0.02,{ }^{* * *} P<0.001$, compared with value for control females. 

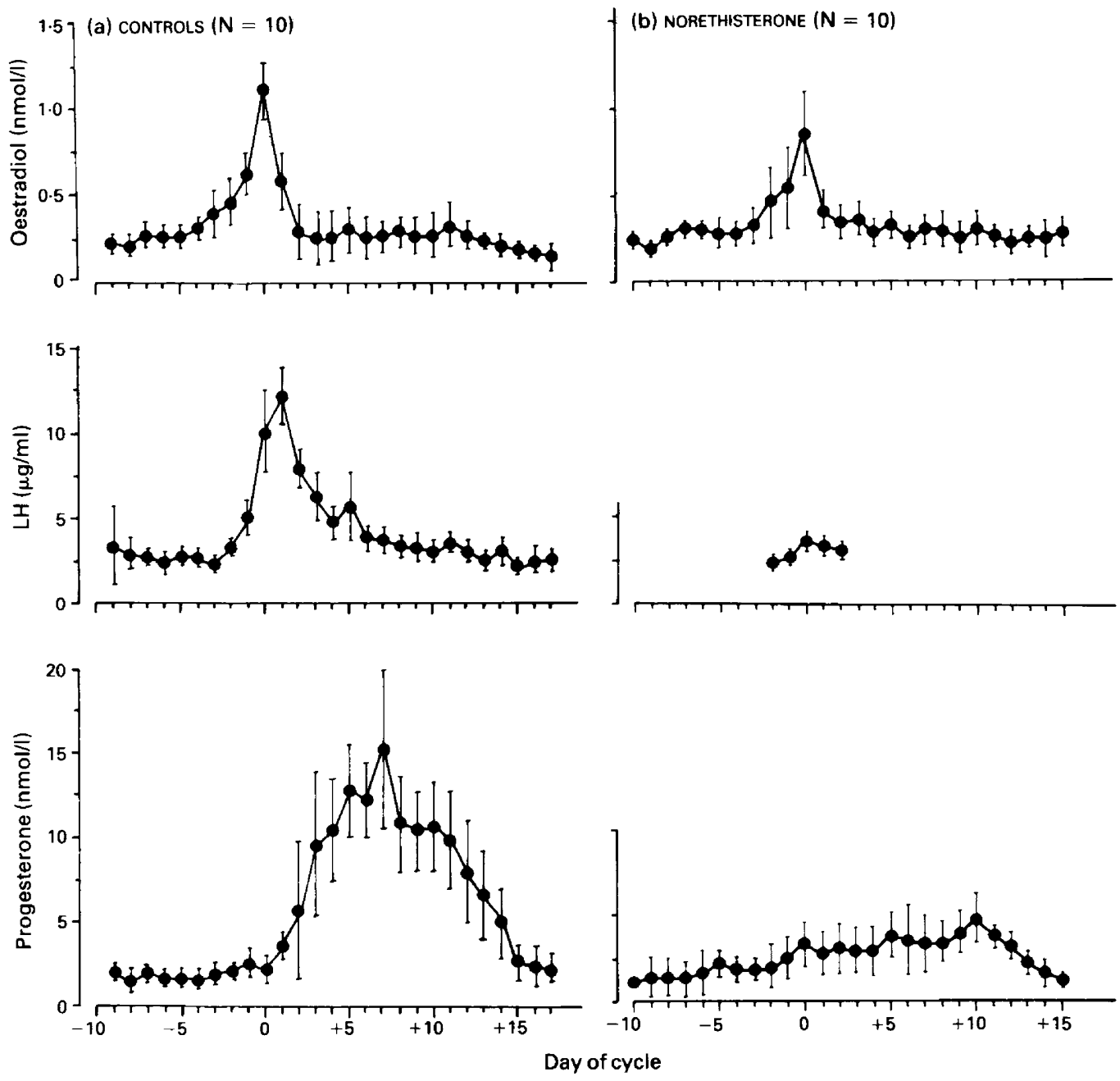

Fig. 1. Changes in concentrations of hormones in (a) control females and (b) anovulatory cycles in 10 norethisterone-treated $(9 \mu \mathrm{g} /$ day) female rhesus monkeys. Values are mean \pm s.e.m. and have been normalized with reference to the peak of oestradiol (Day 0 ).

\section{Hormonal changes}

The hormonal profiles in blood samples taken daily throughout the cycle in the solvent-treated controls (Fig. 1a) were not markedly different from those observed in normal ovulatory cycles in monkeys kept in this colony (Anand Kumar et al., 1980b). In all the 17 treated monkeys the pattern and circulating concentrations of oestradiol were similar to those seen in daily blood samples taken throughout the cycle in the controls, indicating that follicular activity was not affected by norethisterone treatment. In the 10 animals that exhibited anovulatory cycles the mid-cycle $\mathbf{L H}$ peak value was markedly reduced and the circulating concentrations of progesterone remained almost at basal levels even during Phase II of the cycle (Fig. lb), which is consistent with the anovulation as revealed by laparoscopy. In the 7 ovulatory cycles observed in the norethisterone-treated females, the hormonal patterns were not markedly different from those of the controls. 


\section{Mean duration of Phases I and II of the menstrual cycle}

The oestradiol peak occurred around Day 9 of the cycle in all the solvent-treated controls and the mean length of Phase II of the cycle was about 17 days. Except in the 4 anovulatory animals, which showed shortened cycles (Group I), the onset of the oestradiol peak was significantly delayed in most of the treated monkeys (Table 1). Phase II of the cycle in all the 17 treated animals was significantly reduced as compared with the controls (Table 1).

\section{Discussion}

The present studies have shown that intranasal administration of norethisterone at a daily dose of $9 \mu \mathrm{g}$ during the periovulatory period inhibited ovulation in 10 out of 17 treated rhesus monkeys. The oestradiol-induced LH surge was also suppressed in these animals and progesterone remained at basal levels. The hormonal patterns in the remaining 7 ovulatory cycles were indistinguishable from those of the controls. The reason for this lack of a uniform effect, in terms of ovulation inhibition, is not known, but essentially similar findings have been reported for women treated with low doses of norethisterone (Landgren \& Diczfalusy, 1980).

The most consistent effect observed in the norethisterone-treated animals was the shortening of the Phase II of the cycle, indicative of a shortened luteal phase. Shortening of the luteal phase of the cycle has been shown to occur spontaneously in monkeys and women (diZerega \& Hodgen, 1981) and such shortened luteal phases are believed to be a result of alterations in the ratios of FSH:LH in the blood during the cycle (Wilks, Hodgen \& Ross, 1976). FSH concentrations were not measured in the present studies because of difficulties in setting up accurate assays for measuring monkey FSH in our laboratories without an appropriate FSH standard. However, since the mid-cycle peak levels of LH were markedly reduced in most of the treated animals, it is likely that FSH:LH ratios were altered. It remains to be shown whether the treatment cycles exhibiting shortened luteal phases are also infertile as has been suggested for naturally occurring cycles with shortened luteal phases in women (diZerega \& Hodgen, 1981).

The pharmacological effects observed in the present study have been well-documented for a number of progestagens (Martinez-Manautou et al., 1966; Fotherby, 1977; Landgren \& Diczfalusy, 1980). What is novel, however, is that these effects were observed by administering much lower doses of the synthetic progestagen $(\bumpeq 2 \mu \mathrm{g}$ per $\mathrm{kg}$ body weight) than currently used for administering norethisterone orally or systemically. The present studies therefore indicate that the intranasal route of drug delivery could be used to advantage for reducing the dose of hormonal contraceptives without a concomitant loss of efficacy.

This work was supported by the World Health Organisation, Geneva, and was carried out during the award of the Jawaharlal Nehru Fellowship to T.C.A.K.

\section{References}

Anand Kumar, T.C., David, G.F.X., Umberkoman, B. \& Saini, K.D. (1974) Uptake of radioactivity by body fluids and tissues in rhesus monkeys after intravenous injection or intranasal spray of tritium-labelled oestradiol and progesterone. Curr. Sci. 43, 435-439.

Anand Kumar, T.C., David, G.F.X., Kumar, K., Umberkoman, B. \& Krishnamoorthy, M.S. (1976) A new approach to fertility regulation by interfering with neuroendocrine pathways. In Neuroendocrine Regulation of Fertility, pp. $314-322$. Ed. T. C. Anand Kumar. S. Karger, Basel.
Anand Kumar, T.C., David, G.F.X. \& Puri, V. (1977) Ovulation in rhesus monkeys suppressed by intranasal administration of progesterone and norethisterone. Nature, Lond. 270, 532-534.

Anand Kumar, T.C., David, G.F.X. \& Puri, V. (1979) Nasal spray contraceptives. In Advances in Reproduction and Regulation of Fertility, pp. 49-55. Ed. G. P. Talwar. Elsevier/North Holland, Biomedical Press, Amsterdam.

Anand Kumar, T.C., Sehgal, A., David, G.F.X., Bajaj, J.S. \& Prasad, M.R.N. (1980a) Effects of intranasal 
administration of hormonal steroids on serum testosterone and spermatogenesis in rhesus monkey Macaca mulatta. Biol. Reprod. 22, 935-940.

Anand Kumar, T.C., David, G.F.X., Sharma, D.N., Puri, C.P., Puri, V., Dubey, A.K., Sehgal, A., Sankaranarayanan, A. \& Pruthi, J.S. (1980b) Reproductive profile of the rhesus monkey with reference to the testing of contraceptives. In Nonhuman Primate models for Study of Human Reproduction, pp. 37-49. Ed. T. C. Anand Kumar. S. Karger, Basel.

Anand Kumar, T.C., David, G.F.X. \& Puri, V. (1980c) Levels of oestradiol and progesterone in the cerebrospinal fluid of rhesus monkey during the menstrual cycle. J. med. Primatol. 9, 222-232.

Anand Kumar, T.C., David, G.F.X., Sankaranarayanan, A., Puri, V. \& Sundaram, K.R. (1982) Pharmacokinetics of progesterone following its systemic or intranasal spraying to ovariectomized rhesus monkeys. Proc. natn. Acad. Sci. U.S.A. 79, 41854189.

diZerega, G.S. \& Hodgen, G.D. (1981) Luteal phase dysfunction in fertility: A sequel to aberrant folliculogenesis. Fert. Steril. 36, 489-499.

Fotherby, K. (1977) Low doses of gestagens as fertility regulating agents. In Regulation of Human Fertility, pp. 283-321. Ed. E. Diczfalusy. Scriptor, Copenhagen.

Gopinath, P.G., Gopinath, G. \& Anand Kumar, T.C. (1978) Target site of intranasally sprayed substances and their transport across the nasal mucosa: a new insight into the intranasal route of drug-delivery. Current Therapeutic Research 23, 596-607.
Hall, P.E. (1978) The World Health Organization programme for the standardization and quality control of radioimmunoassay of hormone in reproductive physiology. Hormone Res. 9, 440-449.

Landgren, B.M. \& Diczfalusy, E. (1980) Hormonal effects of the $300 \mu \mathrm{g}$ norethisterone (NET) minipill. 1 . Daily steroid levels in $\mathbf{4 3}$ subjects during a pretreatment cycle and during the second month of NET administration. Contraception, 21, 87-113.

Martinez-Manautou, J., Cortez, V., Giner, J., Azuar, R., Casasola, J. \& Rudel, H.W. (1966) Low doses of progestogen as an approach to fertility control. Fert. Steril. 17, 49-57.

Puri, C.P., Puri, V., David, G.F.X. \& Anand Kumar, T.C. (1980) Bioactive luteinizing hormone in the plasma and cerebrospinal fluid of female rhesus monkeys. $J$. med. Primatol. 9, 39-49.

Weick, R.J., Dierschke, D.J., Karsch, F.J., Butler, W.R., Hotchkiss, J. \& Knobil, E. (1973) Periovulatory time courses of circulating gonadotropin and ovarian hormones in the rhesus monkey. Endocrinology 93, 1140-1147.

Wilks, J.W., Hodgen, G.D. \& Ross, G.J. (1976) Luteal phase defects in the rhesus monkey: the significance of serum FSH:LH ratios. J. clin. Endocr. Metab. 43, 1261-1267. 